\title{
PET/MRI Dual-Modality Tumor Imaging Using Arginine-Glycine-Aspartic (RGD)-Conjugated Radiolabeled Iron Oxide Nanoparticles
}

\author{
Ha-Young Lee*1, Zibo Li*1, Kai Chen ${ }^{1}$, Andrew R. Hsu ${ }^{1}$, Chenjie $\mathrm{Xu}^{2}$, Jin Xie ${ }^{2}$, Shouheng $\mathrm{Sun}^{2}$, and Xiaoyuan Chen ${ }^{1}$ \\ ${ }^{I}$ Molecular Imaging Program at Stanford (MIPS), Department of Radiology and Bio-X Program, Stanford University School of Medicine, \\ Stanford, California; and ${ }^{2}$ Department of Chemistry, Brown University, Providence, Rhode Island
}

The purpose of this study was to develop a bifunctional iron oxide (IO) nanoparticle probe for PET and MRI scans of tumor integrin $\alpha_{v} \beta_{3}$ expression. Methods: Polyaspartic acid (PASP)-coated IO (PASP-IO) nanoparticles were synthesized using a coprecipitation method, and particle size and magnetic properties were measured. A phantom study was used to assess the efficacy of PASP-IO as a T2-weighted MRI contrast agent. PASP-IO nanoparticles with surface amino groups were coupled to cyclic arginine-glycine-aspartic (RGD) peptides for integrin $\alpha_{v} \beta_{3}$ targeting and macrocyclic 1,4,7,10-tetraazacyclododecane$N, N^{\prime}, N^{\prime \prime}, N^{\prime \prime \prime}$,--tetraacetic acid (DOTA) chelators for PET after labeling with ${ }^{64} \mathrm{Cu}$. IO nanoparticle conjugates were further tested in vitro and in vivo to determine receptor targeting efficacy and feasibility for dual PET/MRI. Results: PASP-IO nanoparticles made by single-step reaction have a core size of $5 \mathrm{~nm}$ with a hydrodynamic diameter of $45 \pm 10 \mathrm{~nm}$. The saturation magnetization of PASP-IO nanoparticles is about $117 \mathrm{emu} / \mathrm{g}$ of iron, and the measured $r_{2}$ and $r_{2}{ }^{*}$ are 105.5 and $165.5(\mathrm{~s} \cdot \mathrm{mM})^{-1}$, respectively. A displacement competitive binding assay indicates that DOTAIO-RGD conjugates bound specifically to integrin $\alpha_{\mathrm{v}} \beta_{3}$ in vitro. Both small-animal PET and T2-weighted MRI show integrinspecific delivery of conjugated RGD-PASP-IO nanoparticles and prominent reticuloendothelial system uptake. Conclusion: We have successfully developed an IO-based nanoprobe for simultaneous dual PET and MRI of tumor integrin expression. The success of this bifunctional imaging approach may allow for earlier tumor detection with a high degree of accuracy and provide further insight into the molecular mechanisms of cancer.

Key Words: PET; MRI; iron oxide nanoparticle; RGD peptide; bifunctional probe; integrin $\alpha_{\mathrm{v}} \beta_{3}$

J Nucl Med 2008; 49:1371-1379

DOI: 10.2967/jnumed.108.051243

\footnotetext{
Received Oct. 30, 2007; revision accepted Apr. 3, 2008.

For correspondence or reprints contact: Xiaoyuan Chen, Molecular Imaging Program at Stanford (MIPS), Department of Radiology and Bio-X Program, Stanford University School of Medicine, 1201 Welch Rd., P095, Stanford, CA 94305-5484.

E-mail: shawchen@stanford.edu

${ }^{*}$ Contributed equally to this work.

COPYRIGHT () 2008 by the Society of Nuclear Medicine, Inc
}

$\mathbf{P}$ signals emitted by positron-emitting radiotracers to construct images of tracer distribution in vivo $(1,2)$. Recent advances in hardware scanner technology have made it possible to build imaging devices with spatial resolutions greater than $2 \mathrm{~mm}$, thus making it possible to image radiotracers in small-animal models $(3,4)$. However, it still may not be possible to accurately localize an area of increased activity using PET images alone because of the absence of identifiable anatomic structures, particularly in the abdomen $(5,6)$. Researchers have recognized this limitation in oncology imaging and have made attempts over the past decade at developing algorithms to coregister functional and anatomic information with varying levels of success $(7,8)$. Beyer et al. (7) first described the prototype PET/CT scanner used in clinical imaging that precisely and simultaneously coregisters functional data from PET and anatomic images from CT. Although the functional resolution restrictions of $\mathrm{PET}$ and $\mathrm{PET} / \mathrm{CT}$ remain the same, the addition of CT anatomic imaging greatly aids in the accurate localization of regions of increased activity on $\operatorname{PET}(9,10)$.

Although simultaneous PET/CT is already being used on a routine basis in clinical oncology $(7,9)$, the combination of PET with MRI may also offer several advantages. The greatest advantage of performing combined PET/MRI is that it should theoretically be possible to obtain "perfect" spatial registration of molecular/functional PET and anatomic/ functional MRI $(11,12)$. In addition to accurate functional and anatomic localization, highly accurate image registration offers the possibility of using MR images to correct for PET partial-volume effects and aid in PET image reconstruction. Spatial registration of independently acquired PET and MR images is currently performed retrospectively, and recent techniques can partially account for nonrigid tissue deformation that may occur between the 2 image acquisitions $(13,14)$. Incorporation of PET and MRI scanners into a single device would keep subject motion and tissue deformation between image acquisitions to a minimum. Compared with PET/CT, PET/MRI also has the advantage of greatly reduced 
radiation exposure. Currently, the compatibility of PET detectors with magnetic fields still poses a technical challenge, with space limitations inside the magnet that need to be resolved. However, there have already been prototype PET/MRI systems successfully implemented for smallanimal imaging (15-17).

We believe that the future of MRI-compatible PET scanners (PET/MRI) will greatly benefit from the use of bifunctional nanoprobe conjugates. In the current study, we developed polyaspartic acid (PASP)-coated iron oxide (IO) nanoparticles conjugated with cyclic arginine-glycineaspartic (RGD) peptides and the macrocyclic chelating agent 1,4,7,10-tetraazacyclododecane- $N, N^{\prime}, N^{\prime \prime}, N^{\prime \prime \prime}$, -tetraacetic acid (DOTA) for integrin $\alpha_{\mathrm{v}} \beta_{3}$ recognition and positronemitting radionuclide ${ }^{64} \mathrm{Cu}$ (half-life $\left[\mathrm{t}_{1 / 2}\right]=12.7 \mathrm{~h}$ ) labeling. Overall, we have demonstrated the applicability and efficacy of these iron oxide-RGD nanoprobes for dual PET/MRI of tumor integrin $\alpha_{v} \beta_{3}$ expression in vivo using a small-animal model (Fig. 1).

\section{MATERIALS AND METHODS}

Ferric chloride hexahydrate $\left(\mathrm{FeCl}_{3} \cdot 6 \mathrm{H}_{2} \mathrm{O} \geq 98 \%\right)$, ferrous chloride tetrahydrate $\left(\mathrm{FeCl}_{2} \cdot 4 \mathrm{H}_{2} \mathrm{O} \geq 98 \%\right)$, 1-ethyl-3-[3-(dimethylamino)propyl]carbodiimide (EDC), $\mathrm{N}$-hydroxysulfonosuccinimide (SNHS), and Chelex 100 resin (50-100 mesh) were purchased from Aldrich. Ammonium hydroxide solution (28\%) was obtained from Fisher Scientific, and polyaspartic acid (average molecular weight [MW], 2,000-3,000 g/mol) was obtained from LANXESS Co. DOTA was purchased from Macrocyclics, Inc., and NHSpoly(ethylene glycol) (PEG)-maleimide (MAL) (MW, 3,400) was purchased from Nektar. Water and all buffers were passed over a Chelex 100 column $(1 \times 15 \mathrm{~cm})$ before use in radiolabeling procedures. Thiolated RGD peptide $\mathrm{c}(\mathrm{RGD}(\varepsilon$-acetylthiol)K) (RGD-SH) was prepared by following a previously reported procedure (18). ${ }^{64} \mathrm{Cu}\left(\mathrm{t}_{1 / 2}=12.7 \mathrm{~h}\right)$ was obtained from the University of WisconsinMadison, and PD-10 desalting columns were purchased from GE Healthcare. Female athymic nude mice (age, 4-5 wk) were obtained from Harlan.

\section{Preparation of PASP-Coated IO Nanoparticles}

To prepare PASP-coated IO nanoparticles, PASP (0.8 g, 0.3 mmol) was dissolved in ammonia $(4 \mathrm{M}, 2.5 \mathrm{~mL})$; the resulting solution was added to $6 \mathrm{~mL}$ of $0.6 \mathrm{M} \mathrm{FeCl}_{3} \cdot 6 \mathrm{H}_{2} \mathrm{O}$ and $6 \mathrm{~mL}$ of $0.3 \mathrm{M}$ $\mathrm{FeCl}_{2} \cdot 4 \mathrm{H}_{2} \mathrm{O}$ mixture dropwise at $100^{\circ} \mathrm{C}$ under an argon atmosphere. The reaction mixture was stirred for $1 \mathrm{~h}$ at $100^{\circ} \mathrm{C}$, and the color of the solution turned from yellow to black immediately, indicating the formation of iron oxide. PASP-coated IO nanoparticles were then neutralized with dilute $\mathrm{HCl}(0.1 \mathrm{~N})$ to a $\mathrm{pH}$ of 7 . The resultant solution was dialyzed against distilled water with dialysis membrane (molecular weight cutoff [MWCO], 10,000) for $3 \mathrm{~d}$ to remove unreacted PASP and iron salts. Any large particles were removed by centrifuging at $1,500 \mathrm{rpm}$ for $20 \mathrm{~min}$.

\section{Characterization of PASP-Coated IO Nanoparticles}

Nanoparticle size and morphology were examined by using a transmission electron microscope (TEM). TEM micrographs were obtained using a CM 20 microscope (Philips) operated at $200 \mathrm{kV}$. To examine the hydrodynamic diameters of the PASP-coated IO nanoparticles, measurements of dynamic light scattering were performed using a DynaPro molecular sizing instrument (Wyatt Technology Corp.) at $25^{\circ} \mathrm{C}$. The magnetic properties of PASPcoated IO nanoparticles were obtained at room temperature with the magnetic field $\leq 20 \mathrm{kOe}$, using a superconducting quantum

A

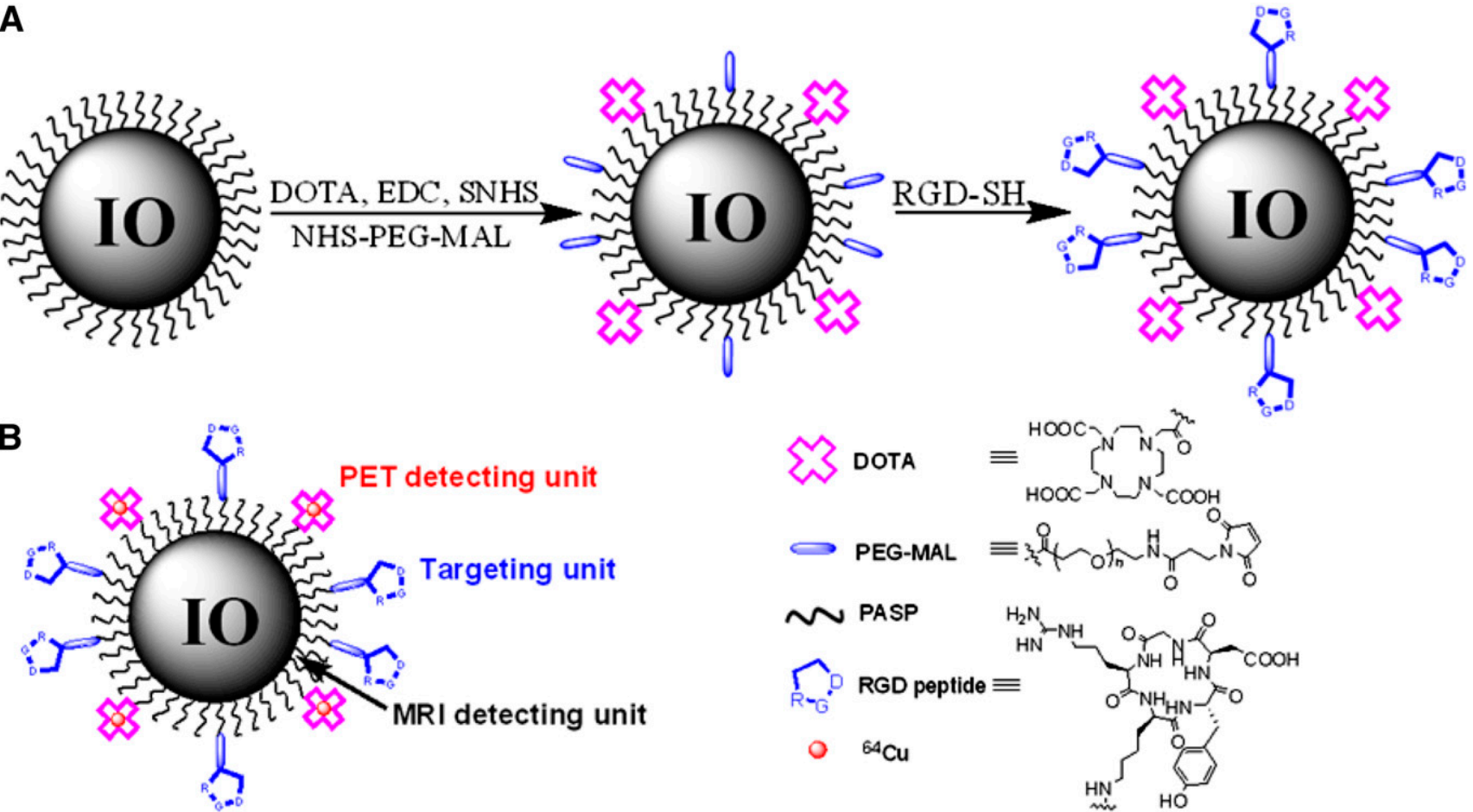

FIGURE 1. (A) Synthesis of PET/MRI dual functional probe DOTA-IO-RGD. DOTA-IO was prepared similarly except that no RGD peptide was used. (B) Illustration of PET/MRI probe based on IO nanoparticle. 
interface device magnetometer (MPMS-XL; Quantum Design). The iron content of the PASP-coated IO nanoparticles was determined by a TJA IRIS Advantage/1000 (Thermo Scientific) radial inductively coupled plasma-atomic emission spectrometer. The number of primary amine groups per IO nanoparticle was determined by ninhydrin assay.

\section{Preparation of DOTA-IO-RGD and ${ }^{64} \mathrm{Cu}$ Radiolabeling}

DOTA was activated according to the reference procedure (19). Briefly, DOTA was activated by EDC and SNHS at pH 5.5 for $30 \mathrm{~min}$ with a DOTA:EDC:SNHS molar ratio of 10:5:4. The activated DOTA $(0.8 \mu \mathrm{mol})$ and a heterobiofunctional linker, NHS-PEGMAL (MW = 3,400, $4.1 \mathrm{mg}, 1.2 \mu \mathrm{mol}$, respectively), were then added into the $200-\mu \mathrm{L}$ IO solution ( $39 \mu \mathrm{mol}$ iron concentration) at a $\mathrm{pH}$ of 8.5 . The mixture was incubated at $4{ }^{\circ} \mathrm{C}$ for $1 \mathrm{~h}$, and RGD-SH (1.0 mg, $1.5 \mu \mathrm{mol}$ ) was added to the solution at a $\mathrm{pH}$ of 7.0. The mixture was incubated overnight, and the unreacted materials were removed through the PD-10 column and dialysis membrane (MWCO, 10,000). DOTA-IO-RGD was radiolabeled by the addition of ${ }^{64} \mathrm{Cu}\left(5 \mu \mathrm{g}\right.$ of DOTA-IO-RGD per millibecquerel of $\left.{ }^{64} \mathrm{Cu}\right)$ in $0.1 \mathrm{~N}$ sodium acetate ( $\mathrm{pH} 6.5$ ) buffer, and the mixture was incubated for $45 \mathrm{~min}$ at $40^{\circ} \mathrm{C} .{ }^{64} \mathrm{Cu}$-DOTA-IO-RGD was then purified using a PD-10 column with phosphate-buffered saline as the mobile phase. The radioactive fractions containing ${ }^{64} \mathrm{Cu}$-DOTA-IO-RGD were collected for further in vitro and in vivo experiments.

\section{Phantom Study}

To confirm the feasibility of PASP-coated IO nanoparticles as an MRI contrast agent, we first prepared the ferrofluids of PASP-IO nanoparticles and ferumoxide (Feridex; AMAG Pharmaceuticals) with varying iron concentrations from $4 \times 10^{-4}$ to $1.25 \times 10^{-5} \mathrm{M}$ in deionized water. Every sample was filled into an arrangement in microfuge tubes (Eppendorf) without air in a plastic rack. The tubes containing samples were embedded in a phantom consisting of tanks filled with $1 \%$ agarose gels to obtain an appropriate image. T2weighted MRI (repetition time/echo time, 3,000/50, 30 flip angle, 14-cm field of view, $256 \times 256$ matrix, 3 -mm slice thickness) was performed using a 1.5-T MRI system (Excite; GE Healthcare).

To measure the relaxivity of PASP-IO, a transverse T2-weighted spin-echo image was acquired using a 3.0-T scanner (Tim Trio MRI; Siemens). Gel preparations in 2-mL vials were placed in a holder for insertion into the 8-channel volume head resonator. The long axis of the vials was parallel to the static magnetic field, and a transverse tomographic plane orientation was used. A gradient-echo acquisition was used with a repetition time of $2,000 \mathrm{~ms}$, an echo time of $1.8 \mathrm{~ms}$, a slice thickness of $12 \mathrm{~mm}$, and a flip angle of $20^{\circ}$. Inplane resolution was $0.88 \mathrm{~mm}$. The normal first-order shim process was applied, and the phantoms were imaged at room temperature $\left(20^{\circ} \mathrm{C}\right)$.

\section{Cell Lines and Animal Model}

The U87MG human glioblastoma cell line was obtained from American Type Culture Collection and cultured under standard condition (20,21). Animal procedures were performed according to a protocol approved by Stanford University Institutional Animal Care and Use Committee. The U87MG tumor model was generated by subcutaneous injections of $5 \times 10^{6}$ cells in $100 \mu \mathrm{L}$ of phosphatebuffered saline into the front flanks of female athymic nude mice (Harlan). The mice were subjected to small-animal PET and MRI studies when the tumor volume reached $100-300 \mathrm{~mm}^{3}$ (3-4 wk after inoculation).

\section{Displacement Competitive Binding Assay}

In vitro integrin $\alpha_{\mathrm{v}} \beta_{3}$-binding affinities and specificities of DOTA-IO-RGD, DOTA-IO, and c(RGDyK) were assessed via a displacement competitive binding assay using ${ }^{125} \mathrm{I}$-echistatin as the integrin $\alpha_{v} \beta_{3}$-specific radioligand on U87MG human glioblastoma cells (19). Experiments were performed with triplicate samples, and the best-fit $50 \%$ inhibitory concentration $\left(\mathrm{IC}_{50}\right)$ values for the U87MG cells were calculated by fitting the data with nonlinear regression (GraphPad Prism; GraphPad Software, Inc.).

\section{In Vivo Small-Animal PET Studies}

PET scans and image analysis were performed using a rodent model scanner (microPET R4; Siemens) as previously reported (19). U87MG tumor mice $(n=3)$ were each injected with $3.7 \mathrm{MBq}$ of ${ }^{64} \mathrm{Cu}$-DOTA-IO-RGD (300 $\mu \mathrm{g}$ of iron per mouse, DOTA-IORGD carrier added) or ${ }^{64} \mathrm{Cu}$-DOTA-IO (300 $\mu \mathrm{g}$ of iron per mouse, DOTA-IO carrier added) via a tail vein. Nonradioactive IO conjugates were added for a total amount of $300 \mu \mathrm{g}$ of iron injected per animal, which is the same amount as used in the MRI scans on separate animals. Five-minute static PET images were acquired at 1 , 4 , and $21 \mathrm{~h}$ after injection, and the images were reconstructed using a 2-dimensional ordered-subsets expectation maximization algorithm. For the receptor-blocking experiment, a U87MG tumor mouse was coinjected with $10 \mathrm{mg}$ of $\mathrm{c}(\mathrm{RGDyK}) / \mathrm{kg}$ of mouse body weight and $3.7 \mathrm{MBq}$ of ${ }^{64} \mathrm{Cu}-\mathrm{DOTA}-\mathrm{IO}-\mathrm{RGD}(300 \mu \mathrm{g}$ of iron per mouse, $n=3$ ), and the 5-min static PET scans were then performed at 1,4 , and $21 \mathrm{~h}$ after injection. For each PET scan, regions of interest (ROIs) were drawn over the tumor, normal tissue, and major organs using vendor software (Pro 5.2.4.0; ASI) on decay-corrected wholebody coronal images. Maximum radioactivity concentration (accumulation) within a tumor or an organ was obtained from mean pixel values within the combined ROI volume and converted to counts/ $\mathrm{mL} / \mathrm{min}$ using a conversion factor. Assuming a tissue density of $1 \mathrm{~g} / \mathrm{mL}$, the ROIs were converted to counts/g/min and then divided by the administered activity to obtain an estimate of tracer accumulation.

\section{In Vivo MRI Studies}

Mice were anesthetized with 1\%-2\% inhaled isoflurane anesthesia (IsoFlo; Abbott Laboratories) in 1:2 $\mathrm{O}_{2}: \mathrm{N}_{2}$, and DOTA-IO, DOTA-IO-RGD, and DOTA-IO-RGD plus a blocking dose of $\mathrm{c}(\mathrm{RGDyK})(10 \mathrm{mg} / \mathrm{kg})$ were then injected intravenously through a tail vein (300 $\mu \mathrm{g}$ of iron per mouse). An MRI scan was performed using a 3.0-T whole-body clinical scanner (Systems Revision 12.0 M5; GE Healthcare) at $4 \mathrm{~h}$ after injection. The MRI frame consisted of a nonmagnetic stereotactic wrist coil with a cylindric surface coil (5-cm internal diameter) positioned directly over the mouse neck. T2-weighted fast spin-echo imaging was performed under the following conditions: receiver bandwidth, $\pm 16 \mathrm{kHz}$; repetition time, 5,000 ms; echo time, $86 \mathrm{~ms}$; flip angle, $90^{\circ}$; echo train length, 8 ; field of view, $4 \times 4 \mathrm{~cm}$; section thickness, $1 \mathrm{~mm}$, 16 slices; matrix, $256 \times 256$; and scan time, 5 min $25 \mathrm{~s}$. MR images were acquired either perpendicular to the anterior-posterior (long) axis of the animal (coronal) or parallel to the anterior-posterior direction (axially). Signal intensities were measured in defined ROIs, which were in similar locations within the tumor center, using software (Image J; U.S. National Institutes of Health).

\section{Histologic Examination}

The tumor-bearing mice were sacrificed immediately after the completion of MRI scans at 4-h time points. Liver, spleen, tumor, muscle, and kidneys were collected and placed into optimal-cutting- 
temperature compound using a plastic mold, and the samples were immediately frozen using dry ice and placed into a $-80^{\circ} \mathrm{C}$ freezer. Tissue sections were cut into $10-\mu \mathrm{m}$-thick slices and stained with Prussian blue.

\section{Statistical Analysis}

Quantitative data were expressed as mean \pm SD. Means were compared using 1-way ANOVA and Student $t$ test. $P$ values less than 0.05 were considered statistically significant.

\section{RESULTS}

\section{Chemistry}

PASP has 2 kinds of functional groups: carboxylates $(-\mathrm{COOH})$ and amines $\left(-\mathrm{NH}_{2}\right)$. Therefore, IO nanoparticles were coated with PASP through the carboxyl group and the remaining amine group could be used for DOTA or heterolinker conjugation with NHS-PEG-MAL. PASP-coated IO nanoparticles were synthesized using a coprecipitation method and functionalized as shown in Figure 1. TEM reveals that the average size of IO nanoparticles is approximately $5 \mathrm{~nm}$ (Figs. 2A and 2C). A selected area diffraction pattern shows that the IO nanoparticles are magnetite $\left(\mathrm{Fe}_{3} \mathrm{O}_{4}\right)$ (Fig. 2B). In solution, the colloidal particles have a diameter of $45 \pm$ $10 \mathrm{~nm}$ as determined from dynamic light-scattering data. These data show that the colloidal particles consist of IO crystals covered with a PASP layer. The hysteresis loop of PASP-coated IO (Fig. 2D) has no coercive force showing superparamagnetic behavior, and the saturation magnetization of PASP-coated IO nanoparticles is $116.9 \mathrm{emu} / \mathrm{g}$ of iron compared with $70 \mathrm{emu} / \mathrm{g}$ of iron for ferumoxide. We also performed ninhydrin assay to determine the primary amines available on the surface of the nanoparticles (19). After reaction, the absorbance of the solution was measured on a UV-Vis spectrometer (Varian) at $570 \mathrm{~nm}$. The concentration of the primary amines was read from the calibration curve and converted into primary amino content in the nanoparticle using lysine as standard. It is estimated that each PASP-coated IO has around 65 amino group on the surface.

The amine-modified IO was conjugated to a heterobifunctional cross-linker, NHS-PEG-MAL, for conjugation of RGD-SH. The active NHS ester reacted with the amine group of PASP-coated IO, and the maleimide of PEG reacted with RGD-SH (Fig. 1). DOTA was activated with SNHS/ EDC, and the resulting DOTA-OSSu ester was conjugated with the amine group of PASP-coated IO nanoparticles. All of the products were purified through a PD-10 column and dialysis membrane (MWCO, 10,000). On the basis of the DOTA-OSSu ester-to-NHS-PEG-MAL reaction ratio, we could estimate that each particle has around 35 RGD groups and 30 DOTA chelating units.

Measured $\zeta$-potential of PSAP-IO nanoparticles in water is about $-50 \mathrm{mV}$. The negative charge is attributed to the unreacted PSAP carboxylic groups. Although most of them were anchored onto the particle surface during synthesis and limited the particle growth, leftover carboxylic groups help stabilize the particles against agglomeration and precipita-
FIGURE 2. (A) TEM image of PASPcoated IO nanoparticle. (B) Selected-area electron diffraction pattern of PASP. coated IO nanoparticle. (C) High-resolution TEM image of PASP-coated IO nanoparticle. (D) Magnetization curve of PASPcoated IO nanoparticle.
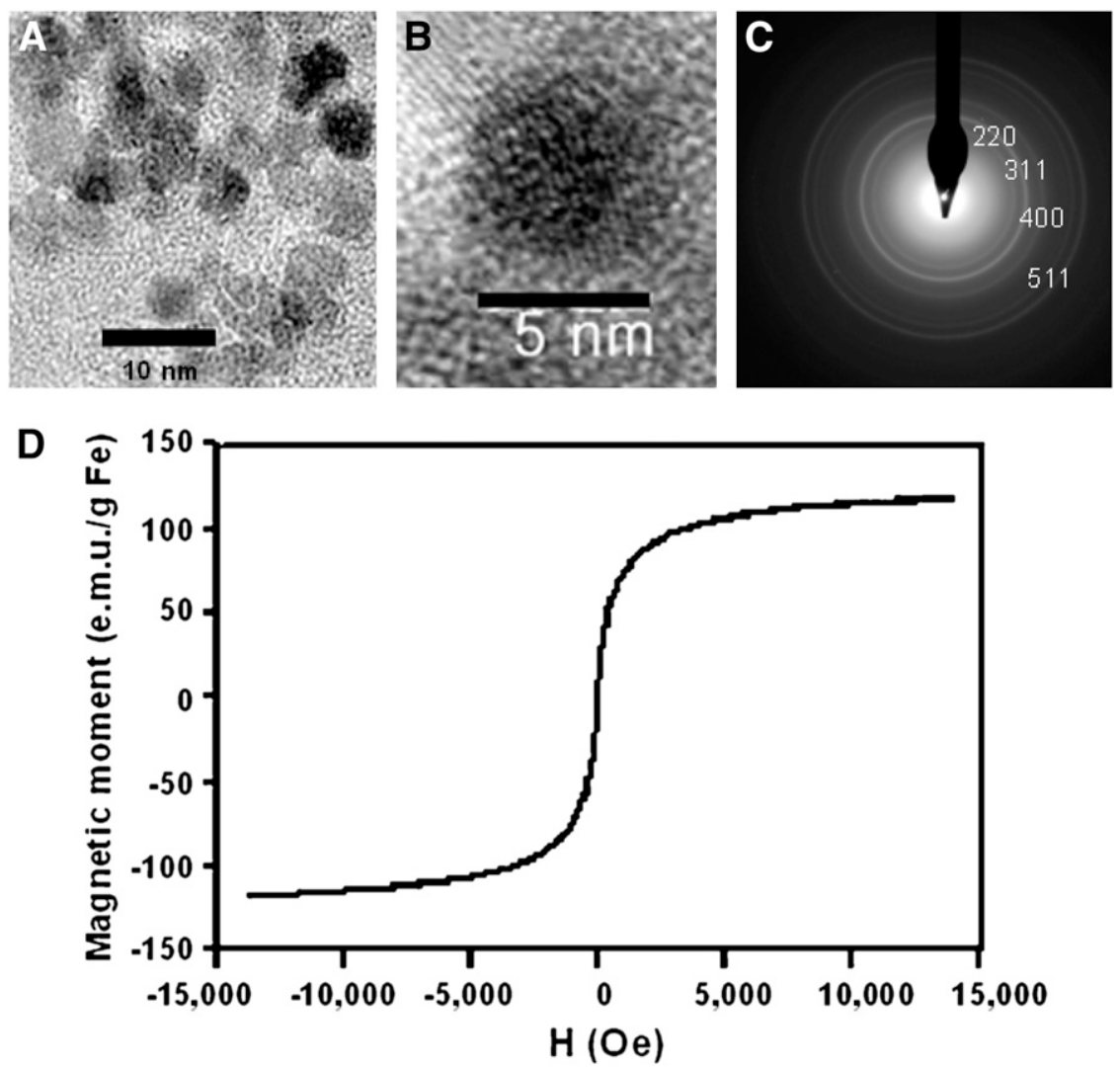
tion. In the following step, the particles were subjected to conjugation with DOTA and RGD peptide, where NHS-PEGMAL was added into the system as a bifunctional linker. PEG was introduced as it would further stabilize the particles in a physiologic environment and potentially help reduce the reticuloendothelial system (RES) uptake.

\section{Phantom Study}

The signal contrast-enhancement effect of T2- and T1weighted MR images of PASP-IO and ferumoxide in a sameconcentration gradient in distilled water ranging from $4 \times$ $10^{-4}$ to $1.25 \times 10^{-5} \mathrm{M}$ iron concentration is shown in Figure $3 \mathrm{~A}$. The graph of signal intensity values converted by the image analysis tool for quantitative measurement is shown in Figure 3B. T2 signal intensity of PASP-IO is reduced faster than that of ferumoxide at the lower iron concentration, and $\mathrm{T} 1$ signal intensity is increased faster than that of ferumoxide at the lower concentration, showing that PASP-IO could be used as both a T2 and a T1 contrast agent.

Transverse T2-weighted spin-echo images were acquired using a 3.0-T Tim Trio MR scanner (Siemens). A gradientecho acquisition was used with a repetition time of 2,000 ms, an echo time of $1.8 \mathrm{~ms}$, a slice thickness of $12 \mathrm{~mm}$, and a flip angle of $20^{\circ}$. The normal first-order shim process was applied, and the phantoms were imaged at room temperature $\left(20^{\circ} \mathrm{C}\right)$. The measured $r_{2}$ and $r_{2}{ }^{*}$ were 105.5 and 165.5 $\mathrm{mM}^{-1} \mathrm{~s}^{-1}$, respectively (Fig. 3B), which were somewhat lower than those of ferumoxide $\left(r_{2}, 151.9 \mathrm{mM}^{-1} \mathrm{~s}^{-1}\right.$, and $r_{2}{ }^{*}$, $\left.275.0 \mathrm{mM}^{-1} \mathrm{~s}^{-1}\right)$.

\section{Displacement Competitive Binding Assay}

Nanoparticles conjugated with RGD must show high binding affinity with integrin $\alpha_{\mathrm{v}} \beta_{3}$ to be used as tumor targeting agents. Receptor-binding affinity studies of DOTA-IO-RGD for $\alpha_{v} \beta_{3}$ integrin were performed using $\alpha_{v} \beta_{3}$-positive U87MG cells. Monomeric RGD peptide $\mathrm{c}(\mathrm{RGDyK})$ and DOTA-IO were also tested as controls. Figure 4 shows that DOTA-IORGD is able to inhibit ${ }^{125} \mathrm{I}$-echhistatin binding to integrin $\alpha_{\mathrm{v}} \beta_{3}$ expressed on U87MG cells. The $\mathrm{IC}_{50}$ value is $34 \pm$ $5 \mathrm{nM}$ of particle concentration in DOTA-IO-RGD. In the same condition, $\mathrm{c}(\mathrm{RGDyK})$ had an $\mathrm{IC}_{50}$ value of $250 \pm 60$ $\mathrm{nM}$. We did not observe any inhibition effect for the DOTAIO control particle.

\section{In Vivo PET Studies}

DOTA-IO and DOTA-IO-RGD can be labeled with ${ }^{64} \mathrm{Cu}$ at a specific activity of around $185 \mathrm{GBq} / \mathrm{g}$ of iron. Figure 5 shows representative coronal PET images of U87MG-tumorbearing mice at different time points after injecting $3.7 \mathrm{MBq}$ of radiotracer. As $300 \mu \mathrm{g}$ of iron were injected for each MRI scan, we added $280 \mu \mathrm{g}$ of iron-equivalent nonradiolabeled IO particles per $3.7 \mathrm{MBq}$ of ${ }^{64} \mathrm{Cu}$-labeled particles $(20 \mu \mathrm{g}$ of iron) to keep the total amount of particles consistent in these 2 measurements. The U87MG tumor was clearly visualized with high contrast relative to the contralateral background from 1 to $21 \mathrm{~h}$ after injection of ${ }^{64} \mathrm{Cu}$-DOTA-IO-RGD. Compared with the RGD conjugated nanoparticle, the non-
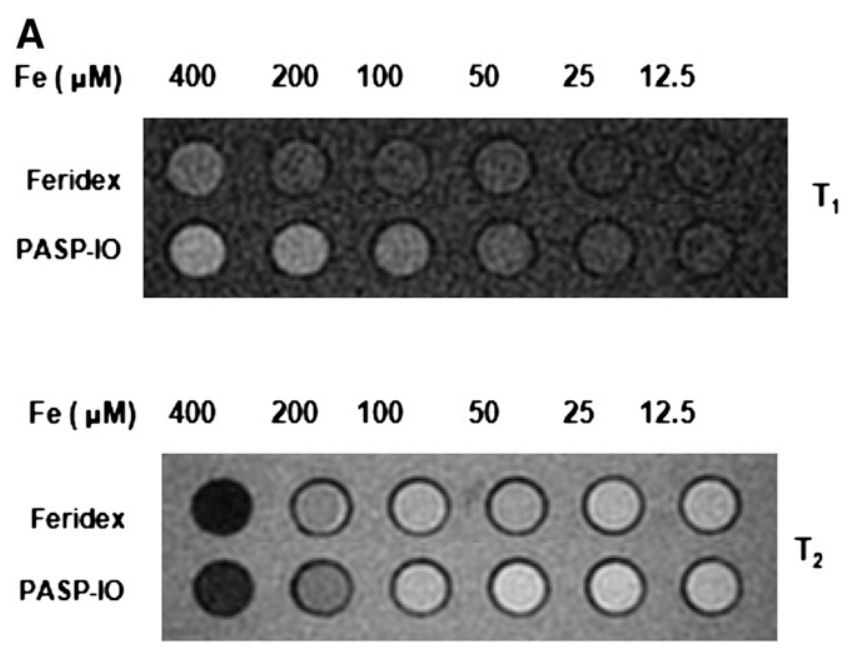

B
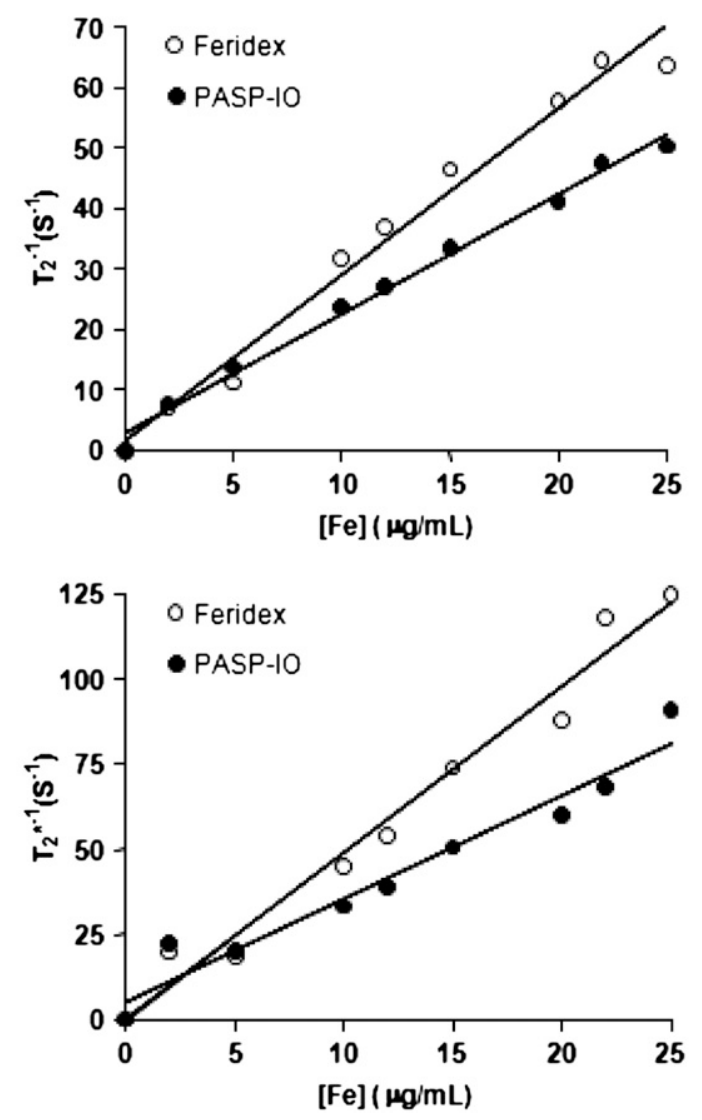

FIGURE 3. (A) Phantom image acquired from T1-weighted MRI scan (top) and T2-weighted MRI scan (bottom) for ferumoxide and PASP-IO at different iron concentrations. (B) 1/T2 (top) and 1/T2* (bottom) vs. Fe concentration for PASP-IO and ferumoxide. Relaxivity values $r_{2}$ and $r_{2}{ }^{*}$ were obtained from slopes of linear fits of experimental data.

targeted particle ${ }^{64} \mathrm{Cu}$-DOTA-IO showed significantly lower tumor uptake. Tumor accumulation at $1 \mathrm{~h}$ after injection of ${ }^{64} \mathrm{Cu}$-DOTA-IO-RGD was $7.9 \pm 0.8$ percentage injected dose per gram $(\% \mathrm{ID} / \mathrm{g})$ and steadily increased and peaked at about $4 \mathrm{~h}$ after injection $(10.1 \pm 2.1 \% \mathrm{ID} / \mathrm{g})$. At $21 \mathrm{~h}$ after injection, tumor uptake slightly decreased to $9.8 \pm 3.2 \%$ ID/ 


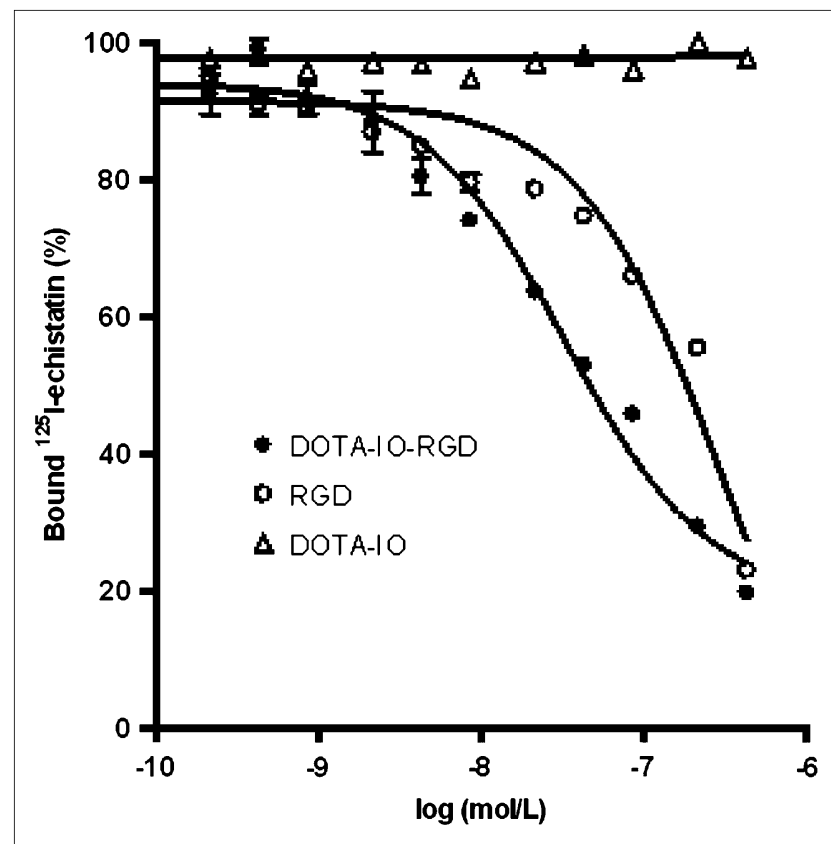

FIGURE 4. Inhibition of ${ }^{125}$-echistatin (integrin $\alpha_{\mathrm{v}} \beta_{3}$-specific) binding to integrin $\alpha_{v} \beta_{3}$ on U87MG cells by DOTA-IO-RGD, $\mathrm{c}(\mathrm{RGDyK})$, and DOTA-IO $(n=3$, mean $\pm \mathrm{SD})$.

g. A blocking experiment with a saturating dose of c(RGDyK) (10 mg/kg of mouse body weight) coinjected with ${ }^{64} \mathrm{Cu}-$ DOTA-IO-RGD revealed a significant reduction in ${ }^{64} \mathrm{Cu}-$ DOTA-IO-RGD uptake. There was no significant difference in liver and kidney uptake between ${ }^{64} \mathrm{Cu}$-DOTA-IO-RGD and ${ }^{64} \mathrm{Cu}$-DOTA-IO at all time points. On the basis of quantitative ROI analysis, both conjugates showed prominent liver uptake $(31.1 \pm 2.5 \% \mathrm{ID} / \mathrm{g}$ at $1 \mathrm{~h}$ after injection, $22.6 \pm$ $2.9 \% \mathrm{ID} / \mathrm{g}$ at $4 \mathrm{~h}$ after injection, and $11.7 \pm 1.2 \% \mathrm{ID} / \mathrm{g}$ at $21 \mathrm{~h}$ after injection) and minimal kidney uptake $(5.1 \pm 0.5 \% \mathrm{ID} / \mathrm{g}$ at $1 \mathrm{~h}$ after injection, $4.9 \pm 0.8 \% \mathrm{ID} / \mathrm{g}$ at $4 \mathrm{~h}$ after injection, and $4.4 \pm 1.0 \% \mathrm{ID} / \mathrm{g}$ at $21 \mathrm{~h}$ after injection), indicating that the majority of injected IO conjugates were mainly taken up by the RES.

FIGURE 5. (A) Decay-corrected wholebody coronal PET images of nude mouse bearing human U87MG tumor at 1, 4, and $21 \mathrm{~h}$ after injection of $3.7 \mathrm{MBq}$ of ${ }^{64} \mathrm{Cu}-$ DOTA-IO, ${ }^{64} \mathrm{Cu}-\mathrm{DOTA}-\mathrm{IO}-\mathrm{RGD}$, or ${ }^{64} \mathrm{Cu}-$ DOTA-IO-RGD with $10 \mathrm{mg}$ of c(RGDyK) peptide per kilogram (300 $\mu \mathrm{g}$ of ironequivalent $\mathrm{IO}$ particles per mouse). (B) Time-activity curves of U87MG tumors after injection of $3.7 \mathrm{MBq}$ of ${ }^{64} \mathrm{Cu}-\mathrm{DOTA}$ IO, ${ }^{64} \mathrm{Cu}$-DOTA-IO-RGD, or ${ }^{64} \mathrm{Cu}-\mathrm{DOTA}$ IO-RGD with blocking dose of c(RGDyK) ( $n=3$ /group).

\section{In Vivo MRI Studies}

To investigate the integrin $\alpha_{\mathrm{v}} \beta_{3}$-targeting ability and MRI visibility of DOTA-IO-RGD conjugates, in vivo T2weighted fast spin-echo MRI was performed with mice bearing U87MG tumors (Fig. 6). As no radioactive animal is allowed at the clinical 3.0-T scanner, we used a group of mice separate from the one used for the PET results for this study. Each mouse was injected via the tail vein with 300 $\mu \mathrm{g}$ of iron, an amount equivalent to that of IO nanoparticles. MRI signal intensity decreased significantly after an injection of DOTA-IO-RGD compared with an injection of DOTA-IO and a coadministration of DOTA-IO-RGD with integrin $\alpha_{\mathrm{v}} \beta_{3}$-blocking agent. Both DOTA-IO and DOTA-IO-RGD showed prominent accumulation in the liver and spleen as indicated by strong negative contrast in these 2 RES organs.

\section{Histologic Analysis}

Uptake of DOTA-IO, DOTA-IO-RGD, and DOTA-IORGD plus free RGD was assessed histologically using Prussian blue staining (Fig. 7). Blue spots were observed in the tumor tissue slices injected with DOTA-IO-RGD, whereas there was no significant uptake for plain particles. Blocking integrin $\alpha_{v} \beta_{3}$ receptors with free RGD effectively reduced the number of blue spots in the tumor slices, showing that the accumulation of DOTA-IO-RGD was specifically mediated by integrin $\alpha_{v} \beta_{3}$ binding. In liver and spleen tissue slices, there were significant particle uptakes for all 3 cases, with no visible difference.

\section{DISCUSSION}

Each molecular imaging modality offers its own unique set of advantages and disadvantages with anatomic, functional, and resolution parameters. With the development of multilabeled imaging probes, the same molecular target can be evaluated with 2 or more different imaging modalities. This allows the strengths of each modality to be combined, thereby improving diagnostic accuracy and providing greater
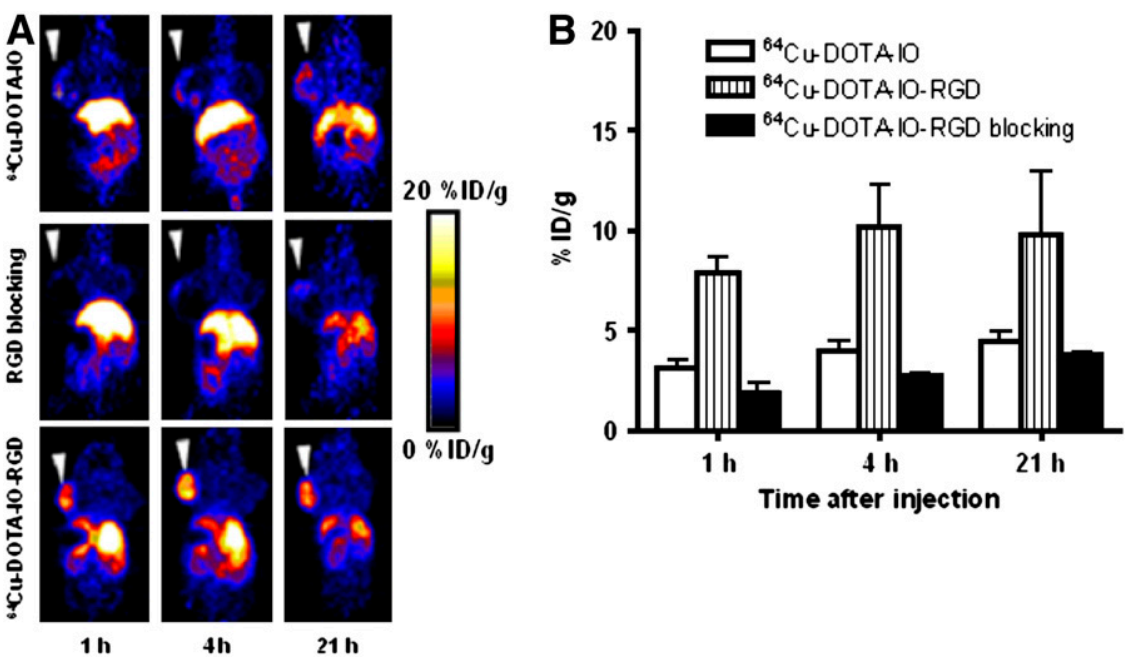

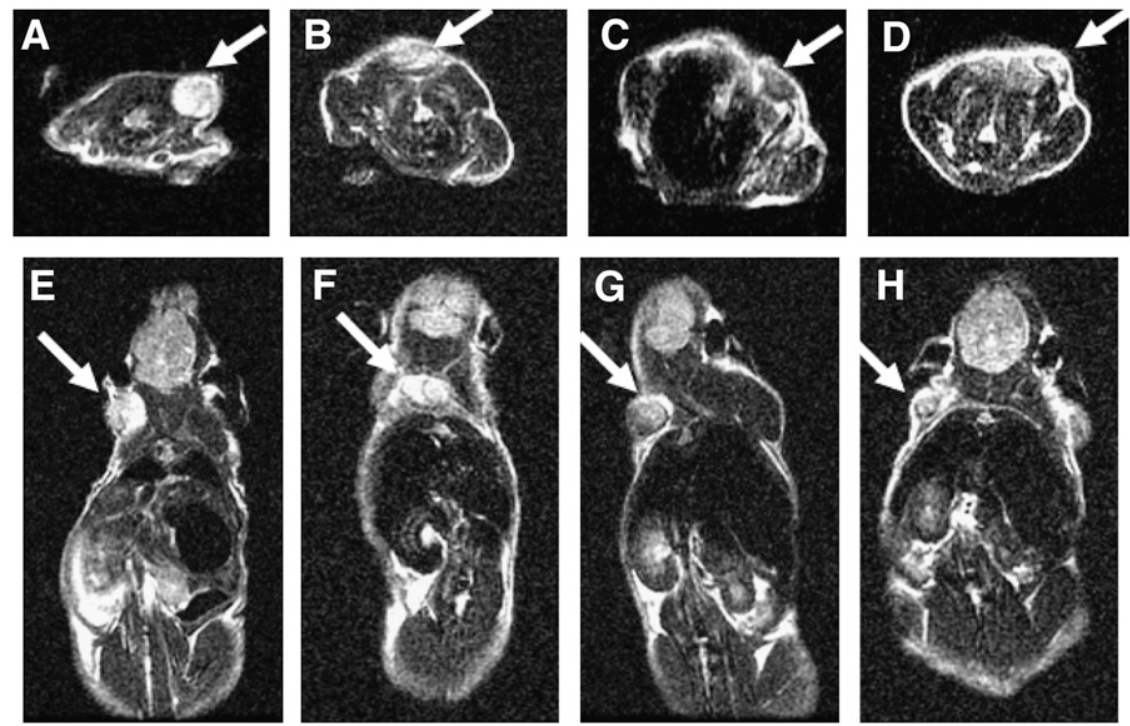

Preinjection

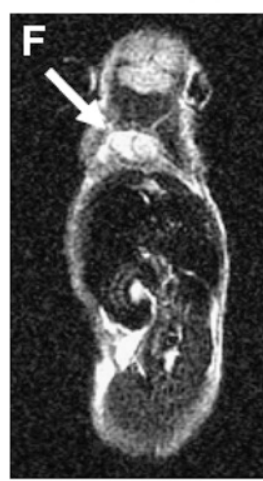

DOTA-IO

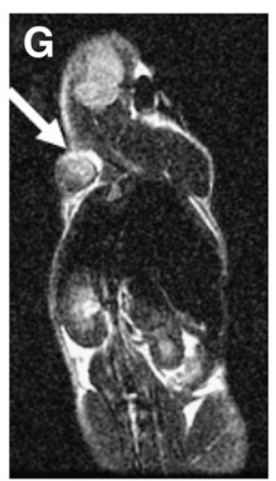

DOTA-IO-RGD

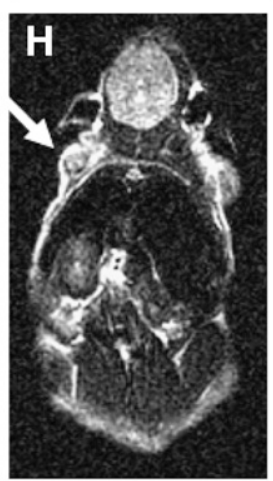

RGD blocking
FIGURE 6. T2-weighted MR images of nude mice bearing U87MG tumor before injection of IO nanoparticles (A and $E$ ) and at $4 \mathrm{~h}$ after tail-vein injection of DOTA-IO (B and F), DOTA-IO-RGD ( $C$ and $G$ ), and DOTA-IO-RGD with blocking dose of $c($ RGDyK) (D and H). insight into underlying disease processes. Although multimodality imaging has been used in current clinical practice to provide a more precise multiparametric description of diseases, it typically uses different imaging agents for each modality. For example, in PET/CT, PET detection uses ${ }^{18} \mathrm{~F}-$ FDG and CT is acquired with or without iodinated contrast medium. Therefore, it would be advantageous to develop a single multifunctional probe that could be detected with more than 1 imaging modality at the same time. With advances in nanotechnology, surface-functionalized nanoparticles may serve as the ideal platform for the construction of multimodality imaging agents (22). To take advantage of the high sensitivity of PET and high spatial resolution of MRI, in this study we developed a MRI/PET bifunctional probe based on IO nanoparticles for dual-modality tumor imaging of integrin $\alpha_{v} \beta_{3}$ expression. RGD peptide was used for targeted delivery of the nanoparticles into integrin $\alpha_{\mathrm{v}} \beta_{3}-$ positive tumors $(20,23,24)$.

In MRI, superparamagnetic IO particles and ultrasmall superparamagnetic iron oxides (USPIOs) coated with dextran and its derivatives have been used to achieve relatively high imaging sensitivity. USPIOs coated with citric acid (25), polystyrene (26), siloxane (27), and polyethylene glycol (PEG) (28) have all been used as MRI contrast agents.
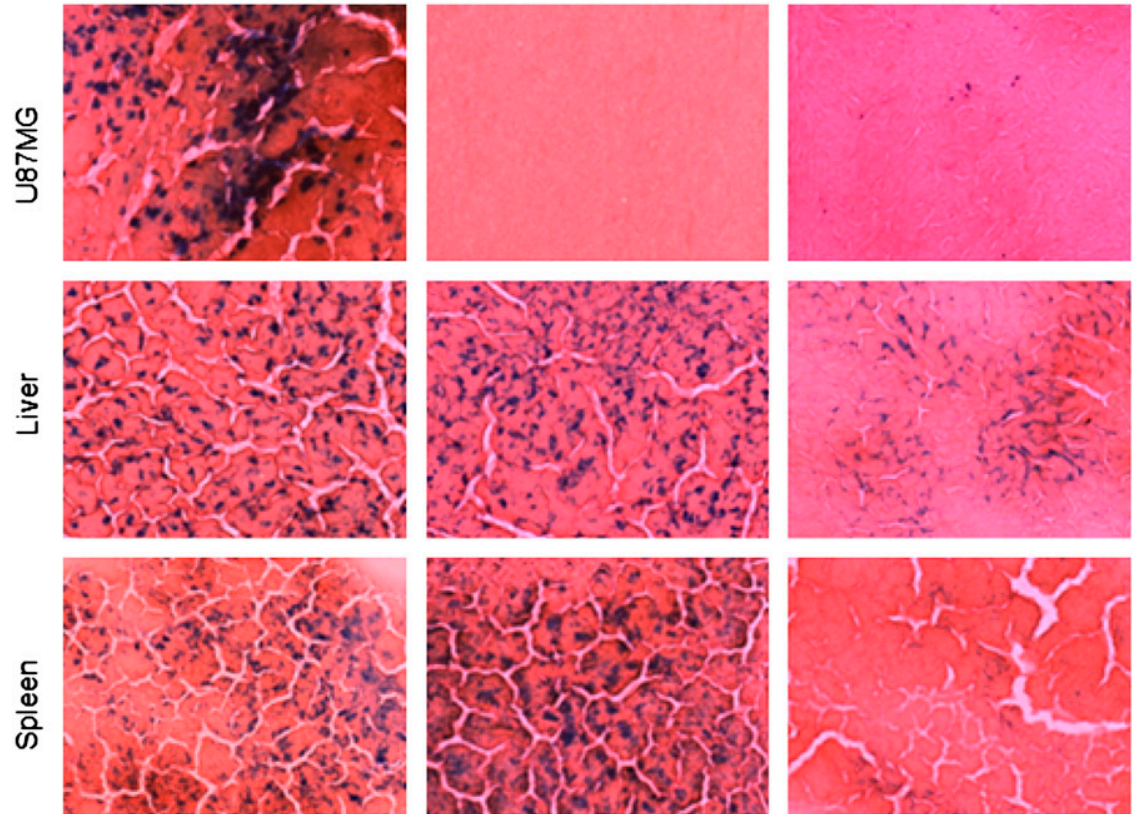

${ }^{64} \mathrm{CU}$-DOTA-IO-RGD

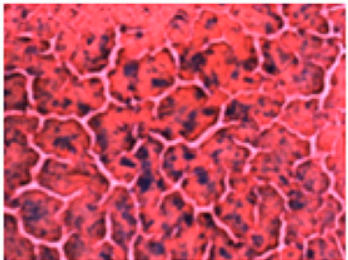

${ }^{64} \mathrm{Cu}-\mathrm{DOTA}-10$

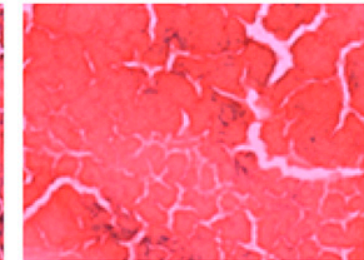

RGD blocking
FIGURE 7. Prussian blue-stained U87MG tumor, liver, and spleen sections after injection of ${ }^{64} \mathrm{Cu}$-DOTA-IO-RGD, ${ }^{64} \mathrm{Cu}$-DOTA-IO, and ${ }^{64} \mathrm{Cu}-\mathrm{DOTA}$-IO-RGD with blocking dose of $\mathrm{c}$ (RGDyK). IO was stained as blue spots in figure (magnification, 200x). 
However, these nanoparticles must go through several steps to achieve surface functionalization. Therefore, we developed a simple method with PASP as the surface-coating material. PASP has low toxicity, biodegradability, and biocompatibility with a peptide chain in which amide linkages extend the chain. In addition, the multifunctional characteristics of the amide linkages afford a variety of modifications by following simple chemical procedures (29). The PASP-IO nanoparticles obtained in this report had a core size of $5 \mathrm{~nm}$, a hydrodynamic diameter of about $45 \mathrm{~nm}$, and a saturation magnetization of about $117 \mathrm{emu} / \mathrm{g}$ of iron. With surface amine groups, the particles were easily functionalized with DOTA for PET isotope chelating and RGD peptide for integrin $\alpha_{v} \beta_{3}$-targeted delivery. DOTA-IO-RGD conjugates were found to bind specifically to integrin $\alpha_{v} \beta_{3}$ in vitro on the basis of displacement competitive binding assays. Subsequent small-animal PET, T2-weighted MRI, and histologic analysis all suggest integrin $\alpha_{\mathrm{v}} \beta_{3}$-specific delivery of the RGD peptide-modified PASP-IO nanoparticles as well as prominent RES uptake of the particles. These correlations demonstrated that the imaging results are an accurate reflection of probe biodistribution.

Overall, the imaging results obtained from different measurement methods are comparable. It has been previously reported that optical imaging/MRI (30) and SPECT/ MRI (31) have been developed. However, the limited tissue-penetration property of optical imaging may limit its clinical application, and compared with SPECT, PET has much higher probe sensitivity. Therefore, PET/MRI is an advantageous combination, and it is critical that multifunctional probe development is improved for the future use of this bifunctional imaging modality. Only qualitative MRI scans and ex vivo Prussian blue staining were performed in this study. Quantification on the basis of MRI might be important to explore the correlations with the results obtained from PET, and it may also be worthwhile to perform PET/MRI coregistration in a future study. Moreover, our results showed that there is still a significant amount of uptake by RES for our IO nanoconstruct. One possibility is the comparatively large hydrodynamic size $(\sim 45 \mathrm{~nm})$, whereas it is more desirable to get the particle size down to $5-10 \mathrm{~nm}$ (32) to achieve more efficient extravasation. Finally, we also observed some nonspecific tumor uptake of the control particles and RGD-IO in the presence of a blocking dose of RGD peptide. More detailed studies are needed in the future to determine whether this is caused by a leaky vasculature or by the uptake of endothelial cells and macrophages in the tumor. In the future, the construction of a fluorescently labeled conjugate for trimodal probe (near-infrared optical, MRI, and PET) may be able to provide even more useful information to molecular mechanisms of disease. Finally, IO has a relatively large surface area for surface conjugation and functionalization. It will be important to explore additional targeting ligands and imaging labels for the development of novel multifunctional contrast agents.

\section{CONCLUSION}

We have designed, synthesized, and tested a novel bifunctional IO-based nanoprobe for dual PET/MRI of tumor integrin $\alpha_{\mathrm{v}} \beta_{3}$ expression. This nanoconstruct has a chelating moiety (DOTA) on the surface for ${ }^{64} \mathrm{Cu}$ labeling (as PET detection motif) and an IO core for MRI. Successful conjugation of this nanoparticle to the integrin $\alpha_{v} \beta_{3}$-binding RGD peptide yielded a tumor-specific probe for multimodality imaging as confirmed by both PET and MRI. The success of this imaging approach may allow for early clinical tumor detection with a high degree of sensitivity while also providing anatomic and molecular information specific to the tumor of interest.

\section{ACKNOWLEDGMENTS}

This work was supported in part by the National Cancer Institute grants R01 CA119053, R21 CA121842, R21 CA102123, P50 CA114747, U54 CA119367, and R24 CA93862; Department of Defense grants W81XWH-07-10374, W81XWH-04-1-0697, W81XWH-06-1-0665, and W81XWH-06-1-0042; and the Korea Research Foundation grant, funded by the Korean government, KRF-2006-352D00061. We also thank the cyclotron teams at University of Wisconsin-Madison for ${ }^{64} \mathrm{Cu}$ production.

\section{REFERENCES}

1. Gambhir SS. Molecular imaging of cancer with positron emission tomography. Nat Rev Cancer. 2002;2:683-693.

2. Weber WA. Use of PET for monitoring cancer therapy and for predicting outcome. J Nucl Med. 2005;46:983-995.

3. Cherry SR, Shao Y, Silverman RW, et al. MicroPET: a high resolution PET scanner for imaging small animals. IEEE Trans Nucl Sci. 1997;44:1161-1166.

4. Tai Y, Ruangma A, Rowland D, et al. Performance evaluation of the microPET focus: a third-generation microPET scanner dedicated to animal imaging. $J$ Nucl Med. 2005;46:455-463.

5. Ruf J, Lopez Hanninen E, Oettle H, et al. Detection of recurrent pancreatic cancer: comparison of FDG-PET with CT/MRI. Pancreatology. 2005;5:266-272.

6. Pannu HK, Cohade C, Bristow RE, Fishman EK, Wahl RL. PET-CT detection of abdominal recurrence of ovarian cancer: radiologic-surgical correlation. Abdom Imaging. 2004;29:398-403.

7. Beyer T, Townsend DW, Brun T, et al. A combined PET/CT scanner for clinical oncology. J Nucl Med. 2000;41:1369-1379.

8. Lavely WC, Scarfone C, Cevikalp $\mathrm{H}$, et al. Phantom validation of coregistration of PET and CT for image-guided radiotherapy. Med Phys. 2004;31:1083-1092.

9. Townsend DW, Beyer T. A combined PET/CT scanner: the path to true image fusion. Br J Radiol. 2002;75(suppl):S24-S30.

10. Beyer T, Townsend DW, Blodgett TM. Dual-modality PET/CT tomography for clinical oncology. Q J Nucl Med. 2002;46:24-34.

11. Cizek J, Herholz K, Vollmar S, Schrader R, Klein J, Heiss WD. Fast and robust registration of PET and MR images of human brain. Neuroimage. 2004;22: 434-442.

12. Myers R. The application of PET-MR image registration in the brain. $\mathrm{Br} \mathrm{J}$ Radiol. 2002;75(suppl):S31-S35.

13. Pluim JP, Maintz JB, Viergever MA. Image registration by maximization of combined mutual information and gradient information. IEEE Trans Med Imaging. 2000;19:809-814.

14. Ge Y, Fitzpatrick JM, Votaw JR, et al. Retrospective registration of PET and MR brain images: an algorithm and its stereotactic validation. J Comput Assist Tomogr. 1994;18:800-810.

15. Shao Y, Cherry SR, Farahani K, et al. Simultaneous PET and MR imaging. Phys Med Biol. 1997;42:1965-1970.

16. Farahani K, Slates R, Shao Y, Silverman R, Cherry S. Contemporaneous positron emission tomography and MR imaging at 1.5 T. J Magn Reson Imaging. 1999;9:497-500. 
17. Higuchi T, Nekolla SG, Jankaukas A, et al. Characterization of normal and infarcted rat myocardium using a combination of small-animal PET and clinical MRI. J Nucl Med. 2007;48:288-294.

18. Xiong Z, Cheng Z, Zhang $\mathrm{X}$, et al. Imaging chemically modified adenovirus for targeting tumors expressing integrin $\alpha_{v} \beta_{3}$ in living mice with mutant herpes simplex virus type 1 thymidine kinase PET reporter gene. J Nucl Med. 2006;47:130-139.

19. Wu Y, Zhang X, Xiong Z, et al. MicroPET imaging of glioma $\alpha_{\mathrm{v}}$-integrin expression using ${ }^{64} \mathrm{Cu}$-labeled tetrameric RGD peptide. J Nucl Med. 2005;46: 1707-1718.

20. Cai W, Shin DW, Chen K, et al. Peptide-labeled near-infrared quantum dots for imaging tumor vasculature in living subjects. Nano Lett. 2006;6:669-676.

21. Chen X, Conti PS, Moats RA. In vivo near-infrared fluorescence imaging of integrin $\alpha_{v} \beta_{3}$ in brain tumor xenografts. Cancer Res. 2004;64:8009-8014.

22. Cai W, Chen X. Nanoplatforms for targeted molecular imaging in living subjects. Small. 2007;3:1840-1854.

23. Montet X, Funovics M, Montet-Abou K, Weissleder R, Josephson L. Multivalent effects of RGD peptides obtained by nanoparticle display. J Med Chem. 2006;49:6087-6093.

24. Zhang C, Jugold M, Woenne EC, et al. Specific targeting of tumor angiogenesis by RGD-conjugated ultrasmall superparamagnetic iron oxide particles using a clinical 1.5-T magnetic resonance scanner. Cancer Res. 2007;67:1555-1562.
25. Taupitz M, Schnorr J, Wagner S, et al. Coronary MR angiography: experimental results with a monomer-stabilized blood pool contrast medium. Radiology. 2002;222:120-126.

26. Bach-Gansmo T. Ferrimagnetic susceptibility contrast agents. Acta Radiol Suppl. 1993;387:1-30.

27. Hahn PF, Stark DD, Lewis JM, et al. First clinical trial of a new superparamagnetic iron oxide for use as an oral gastrointestinal contrast agent in MR imaging. Radiology. 1990;175:695-700.

28. Saeed M, Wendland MF, Engelbrecht M, Sakuma H, Higgins CB. Value of blood pool contrast agents in magnetic resonance angiography of the pelvis and lower extremities. Eur Radiol. 1998;8:1047-1053.

29. Prompruk K, Govender T, Zhang S, Xiong CD, Stolnik S. Synthesis of a novel PEG-block-poly(aspartic acid-stat-phenylalanine) copolymer shows potential for formation of a micellar drug carrier. Int J Pharm. 2005;297:242-253.

30. Sosnovik D, Weissleder R. Magnetic resonance and fluorescence based molecular imaging technologies. Prog Drug Res. 2005;62:83-115.

31. Zielhuis SW, Seppenwoolde JH, Mateus VA, et al. Lanthanide-loaded liposomes for multimodality imaging and therapy. Cancer Biother Radiopharm. 2006; 21:520-527.

32. Choi HS, Liu W, Misra P, et al. Renal clearance of quantum dots. Nat Biotechnol. 2007;25:1165-1170. 